\title{
A case of rhabdomyosarcoma of the right ventricle
}

\author{
RICHARD FIRMIN, ANDREW LOWES, AND BRYAN HICKSON
}

From St Bartholomew's Hospital, London

SUMMARY A patient is described who presented with symptoms suggesting pericarditis and cardiac tamponade, and who was found at necropsy to have a rhabdomyosarcoma of the right ventricle. The presenting features of right ventricular tumours are discussed and some useful pointers to the correct diagnosis suggested.

Primary sarcomas of the heart are rare and difficult to diagnose. Approximately 300 such tumours of varying histological types have been described. We present a case of rhabdomyosarcoma of the right ventricle mimicking pericarditis and cardiac tamponade. In this situation the electrocardiogram may be a useful diagnostic pointer.

\section{Case report}

A 23-year-old housewife was admitted to hospital as an emergency in 1976. She had been quite well until two months earlier when she developed a 'flu-like' illness. Initially her symptoms improved but she subsequently became lethargic, short of breath, and oedematous.

On examination she looked unwell and had a low grade pyrexia. Her pulse was of small volume with obvious paradox. The blood pressure was 100/75 $\mathrm{mmHg}$. The heart was not clinically enlarged but there was a right ventricular heave. A systolic ejection murmur was audible in the pulmonary area and intermittently a pericardial friction rub. Though the lung bases were clear, the jugular venous pressure was raised to the angle of the jaw with a prominent ' $a$ ' wave, the liver was enlarged $6 \mathrm{~cm}$ below the costal margin, and the dependent tissues were oedematous.

Initial investigations showed the erythrocyte sedimentation rate to be $6 \mathrm{~mm}$ in the first hour, the haemoglobin $14.0 \mathrm{~g} / \mathrm{dl}$, and the white cell count $10.9 \times 10^{9} / 1$, with a lymphocytosis. A biochemical screening, including plasma electrophoresis, was normal except for an increase in serum aspartate transaminase to 55 units. Chest $x$-ray film showed a slight increase in the cardiothoracic ratio $(14 / 26)$, with prominence of the right heart border but the lung fields were normal. The electrocardiogram showed sinus rhythm, rate approximately 110 beats per minute, right axis deviation (axis $+120^{\circ}$ ), and a prominent $P$ wave of right atrial form. The $Q R S$ complexes were of small voltage throughout with $\mathrm{rsr}^{\prime}$ configuration in lead $\mathrm{V} 1$ and persistent $\mathrm{S}$ waves over the chest leads. An echocardiogram showed a small posterior pericardial effusion with paradoxical movement of the left ventricular posterior wall.

A diagnosis of pericarditis with effusion was made. Initial treatment with bed rest and diuretics produced some improvement until she had a syncopal attack 10 days after admission. Urgent decompression of the pericardium was recommended. As the effusion was small and posterior and the diagnosis in doubt, a formal left anterior thoracotomy was perfomed. At operation the pericardium was lax, containing only $75 \mathrm{ml}$ clear fluid. The right ventricle and the interventricular septum, however, felt nodular and a biopsy of this area was taken. Her postoperative course was stormy because of low cardiac output causing renal and hepatic failure. She died on the sixteenth postoperative day. The biopsy was reported as showing normal myocardial tissue.

Necropsy showed a large polypoid tumour which almost filled the right ventricular cavity (Fig. 1). The tumour arose from a broad base in the interventricular septum and projected into the tricuspid valve ring. Histological sections showed an infiltrating spindle cell tumour with a myxomatous stroma (Fig. 2a). Cross striations were demonstrated with phosphotungstic acid-haematoxylin staining, consistent with a diagnosis of rhabdomyosarcoma (Fig. 2b). There was no evidence of metastasis.

\section{Discussion}

This case illustrates how ventricular tumours may cause diagnostic difficulty. Systemic upset, fever, pulsus paradoxus, pericardial friction rub, and peri- 


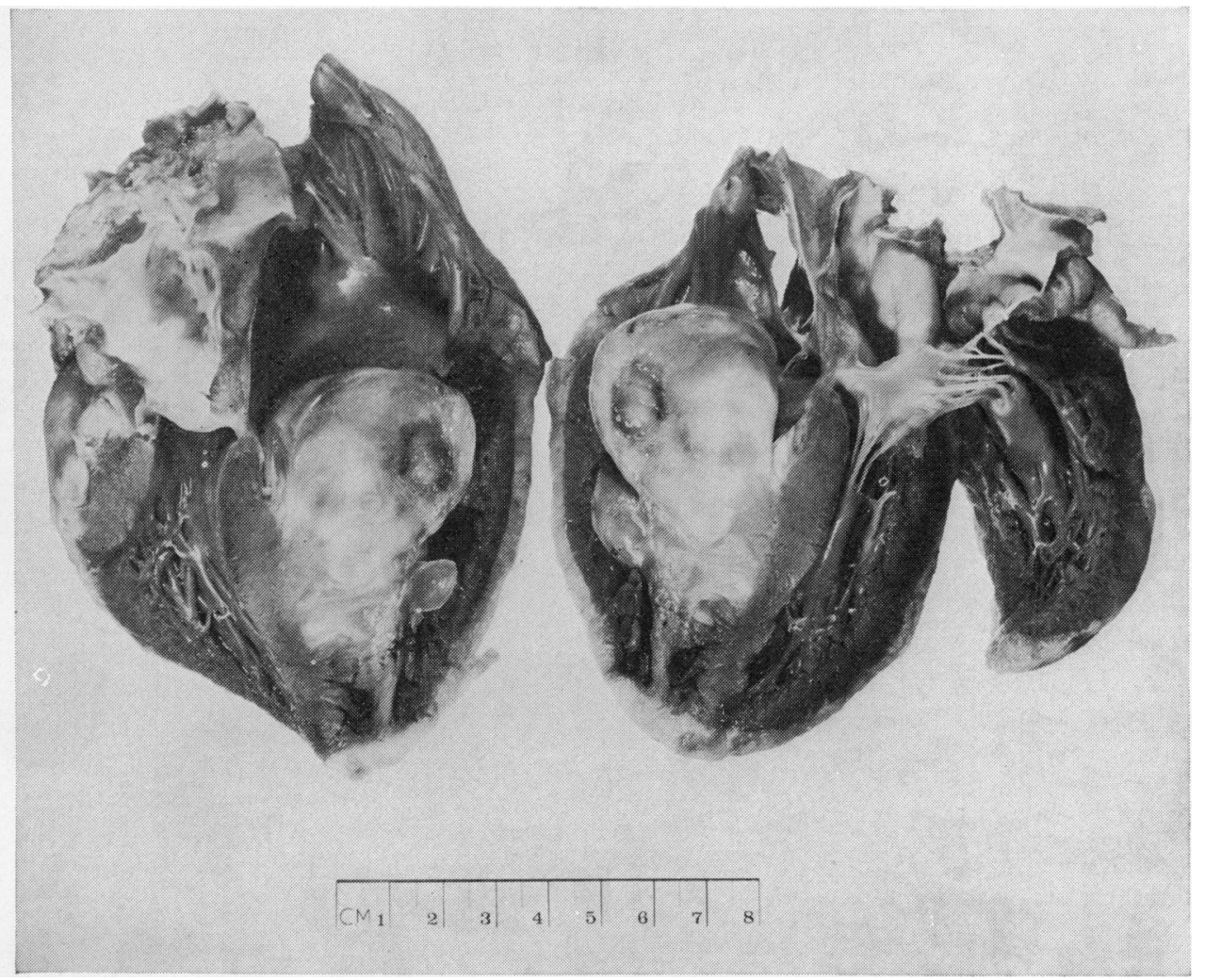

Fig. 1 The heart at necropsy showing a large polypoid tumour arising from the interventricular septum and almost filling the right ventricular cavity. The tumour projects into the tricuspid valve ring. Histologically it was a rhabdomyosarcoma.

cardial effusion are features of both pericarditis and myocardial tumour (Sochocky, 1971). The echocardiogram was not helpful, as a comparatively small effusion was shown, the right ventricular cavity was not seen, and the tumour was missed. Many of the previously reported cases of ventricular tumours were only discovered at necropsy, but the early diagnosis is important as not all tumours are malignant and there is a reported case of survival after surgical removal (Dong et al., 1962). Tumours of atrial origin are mainly myxomas, the presenting features well recognised, and the diagnosis frequently achieved. The features of ventricular tumours are less well known though similar to those of atrial origin. They include breathlessness, chest pain, fever, cough, loss of weight, pleural and pericardial effusions, conduction defects, emboli, murmurs, heart failure, and arrhythmias, the last two being particularly common (Harvey, 1968; Scobbo and Chung, 1974). Many of these tumours are right ventricular in origin and present with puzzling low output right heart failure which responds poorly to treatment (O'Reilly et al., 1975).

The electrocardiogram frequently shows right axis deviation, right bundle-branch block, or right ventricular hypertrophy. Pericardial effusion is common enough to warrant suspicion of intracardiac tumour in any case where the cause is not immediately apparent and the triad of low output heart failure, pericardial effusion, and an electrocardiogram showing right axis deviation or right ventricular hypertrophy of puzzling origin seems to be particularly characteristic. In such cases the most useful investigations are echocardiography and angiography. Cytology of pericardial fluid, which is typically blood stained in cases of cardiac tumour, may occasionally show malignant cells.

Echocardiography in experienced hands is a reliable diagnostic technique for pericardial effusions (Feigenbaum, 1973). It is also a sensitive method of detecting left atrial and ventricular tumours. Right ventricular tumours may be less readily diagnosed but at least one such case has been reported (Sassé 


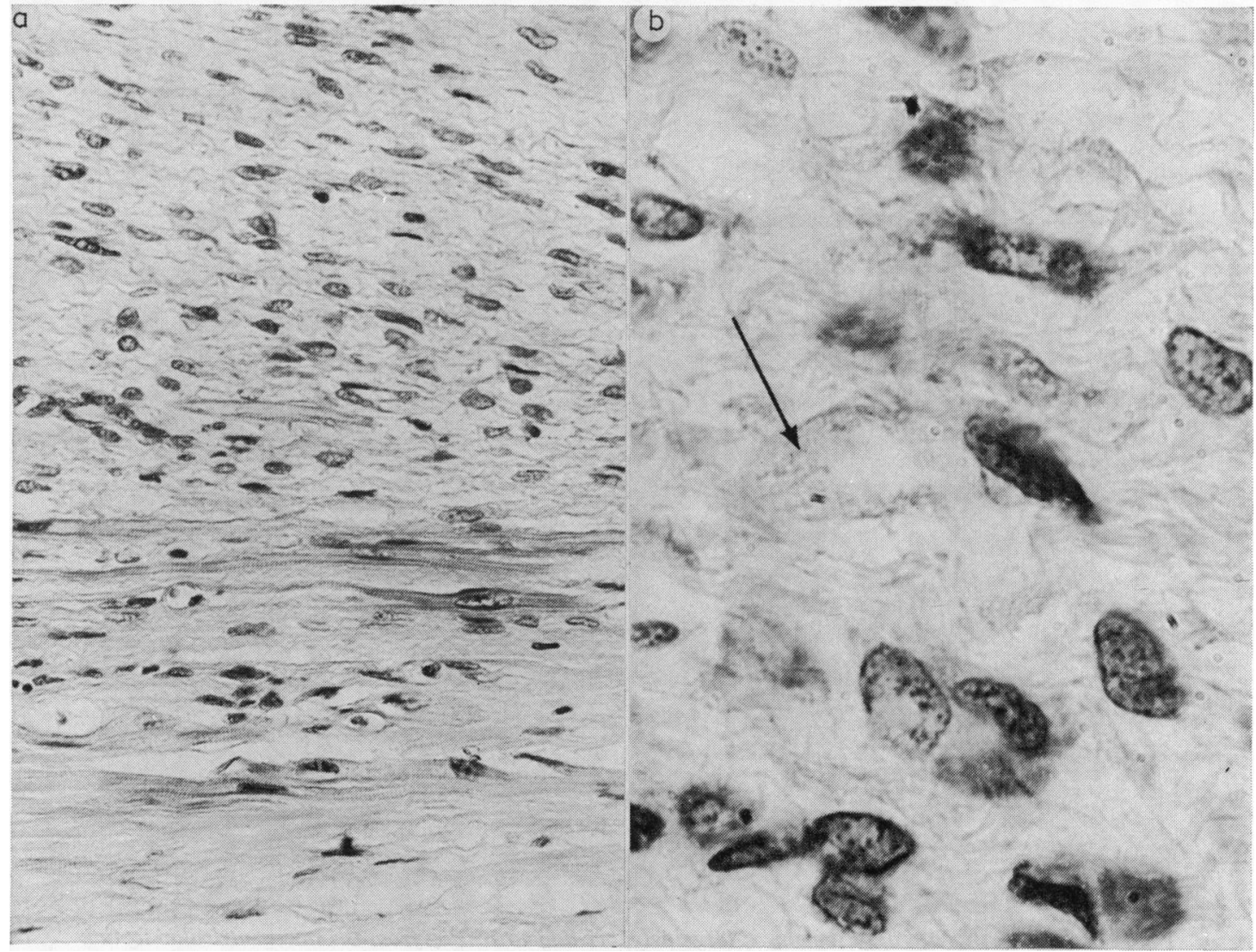

Fig. 2(a) Tumour cells infiltrating normal myocardium (Haematoxylin and eosin. $\times 400).($ b) $A$ high power view showing a tumour cell with cross striations (arrow). (Haematoxylin and eosin. $\times 1600$, oil immersion.)

et al., 1975). It seems a sensible precaution to show clearly all the heart chambers in cases of pericardial effusion or low output heart failure with the features described above. Failure to show the right ventricular cavity should be accepted as important evidence of cavity obliteration.

Paradoxical motion of the ventricular septum and walls is well recognised in a variety of conditions. Paradoxical 'square wave' septal motion has been reported in association with right ventricular tumour (Sassé et al., 1975). Our own case indicates that paradoxical movement of the left ventricular posterior wall may also occur with right ventricular tumour.

It is important that cardiac angiography is undertaken at an early date for it is likely to provide a diagnosis where other methods may fail.

We thank Mr I. M. Hill and the late Dr G. W. Hayward for allowing us to report this patient who was under their care, Dr A. Stansfeld for advice, and $\mathrm{Mr} \mathrm{P}$. Crocker for help in taking the photomicrographs.

\section{References}

Dong, E., Jr., Hurley, E. J., and Shumway, N. E. (1962). Primary cardiac sarcoma. American fournal of Cardiology, 10, 871-878.

Feigenbaum, H. (1973). Echocardiography, pp. 182-183. Lea and Febiger, Philadelphia.

Harvey, W. P. (1968). Clinical aspects of cardiac tumours. American fournal of Cardiology, 21, 328-343.

O'Reilly, M. V., McDonald, R. T., and Fornasier, V. L. (1975). Clinical presentation of a myocardial rhabdomyosarcoma. British Heart fournal, 37, 672-675.

Sassé, L., Lorentzen, D., and Alvarez, H. (1975). Paradoxical septal motion secondary to right ventricular tumor. Fournal of the American Medical Association, 234, 955-956.

Scobbo, R. R., and Chung, E. K. (1974). Cardiac tumours. Postgraduate Medicine, 56, 138-141.

Sochocky, S. (1971). Rhabdomyosarcoma of the heart (a review). Minnesota Medicine, 54, 747-750.

Requests for reprints to Dr Bryan Hickson, St Bartholomew's Hospital, West Smithfield, London EC1A 7BE. 